\title{
Limit Cycles in a Cubic Kolmogorov System with Harvest and Two Positive Equilibrium Points
}

\author{
Qi-Ming Zhang, ${ }^{1}$ Feng Li, ${ }^{2}$ and Yulin Zhao' \\ ${ }^{1}$ College of Science, Hunan University of Technology, Zhuzhou, Hunan 412007, China \\ ${ }^{2}$ College of Science, Linyi University, Linyi, Shandong 276005, China \\ Correspondence should be addressed to Feng Li; lf0539@126.com
}

Received 23 June 2014; Accepted 9 July 2014; Published 20 July 2014

Academic Editor: Tonghua Zhang

Copyright (c) 2014 Qi-Ming Zhang et al. This is an open access article distributed under the Creative Commons Attribution License, which permits unrestricted use, distribution, and reproduction in any medium, provided the original work is properly cited.

A class of planar cubic Kolmogorov systems with harvest and two positive equilibrium points is investigated. With the help of computer algebra system MATHEMATICA, we prove that five limit cycles can be bifurcated simultaneously from the two critical points $(1,1)$ and $(2,2)$, respectively, in the first quadrant. Moreover, the necessary conditions of centers are obtained.

\section{Introduction}

In mathematical ecology, a class of systems of the form

$$
\frac{d x_{i}}{d t}=x_{i} F\left(x_{1}, \ldots, x_{n}\right), \quad i=1, \ldots, n
$$

are frequently used to model the interaction of species occupying the same ecological niche. The differential equations modeling the interaction of two species

$$
\begin{aligned}
& \frac{d x}{d t}=x F(x, y), \\
& \frac{d y}{d t}=y G(x, y),
\end{aligned}
$$

being known as Kolmogorov systems have been studied extensively. It is well known that there is no limit cycles in the classical Lotka-Volterra-Gause model, where $F$ and $G$ are linear. There can of course only be one critical point in the interior of the realistic quadrant $(x>0, y>0)$ in this case, but this can be a centre; however, there are no isolated periodic solutions.

If $F$ and $G$ are quadratic, one might think by analogy that the behavior within the first quadrant is similar to that of a quadratic system. The examples we give show that this is not the case, even when $F$ and $G$ factories. There are many contributions about this system (see $[1,2])$. The latter poses the question whether a predator-prey system can have two or more ecologically stable cycles. If $F$ and $G$ are cubic, there are also many works to consider its limit cycles and dynamics behaviors, see [3, 4]. In [5], the authors discussed a class of cubic Kolmogorov systems with three invariant algebraic curves.

Recently, a system with three positive equilibrium points has been investigated, the authors have investigated the center-focus problems and limit cycles bifurcations. They have proved that each of the two points $(1,2)$ and $(2,1)$ can bifurcate 1 small limit cycle under a certain condition, and 3 limit cycles can occur near point $(1,1)$ at the same step [6]. Other Kolmogorov systems were also investigated recently in $[7,8]$. In this paper, we will consider limit cycles which bifurcate from a class of systems of the form

$$
\begin{aligned}
\frac{d x}{d t}= & -2(2 \beta+\theta)+(8 \beta+5 \theta) x-\frac{7}{2}(2 \beta+\theta) x^{2} \\
& +\frac{1}{2}(4 \beta-\theta) x y+a_{20} x^{3}+\frac{1}{2}\left(6 \beta+3 \theta-4 a_{20}\right) x^{2} y \\
& +\frac{1}{2}\left(-4 \beta-\theta+2 a_{20}\right) x^{2} y,
\end{aligned}
$$




$$
\begin{aligned}
\frac{d y}{d t}= & 2(2 \beta+\theta)-(8 \beta+5 \theta) y+\frac{7}{2}(2 \beta+\theta) y^{2} \\
& +\frac{1}{2}(-4 \beta+\theta) x y+\frac{1}{2}\left(2 b_{20}-4 \beta-\theta\right) x^{3}+b_{20} x^{2} y \\
& +\frac{1}{2}\left(2 \beta-\theta-4 b_{20}\right) x y^{2},
\end{aligned}
$$

which have two positive equilibrium points $(1,1)$ and $(2,2)$. We use our Computer Algebra procedure Mathematic (described in [9]) to compute the focal values at the critical points $(1,1)$ and $(2,2)$. We will show that five limit cycles can bifurcate from points $(1,1)$ and $(2,2)$ simultaneously. Furthermore, some necessary and sufficient conditions for two positive equilibrium points to be centers are also to be given.

This paper is divided into three sections. In Section 2, we use the recursive algorithm to obtain that 5 limit circles could be bifurcated from points $(1,1)$ and $(2,2)$. In Section 3 , necessary and sufficient conditions for two positive equilibrium points to be centers are proved.

\section{Bifurcations of Limit Circles at Two Positive Equilibrium Points}

First of all, it is easy to testify that points $(1,1)$ and $(2,2)$ are two positive equilibriums of system (3) and they are all center or focus. So we need to compute the Lyapunov constants to determine its kind of singular. Now, we consider the point $(1,1)$.

Through the transformations $\tilde{x}=x-1, \tilde{y}=y-1, \tau=\beta t$, we still denote $\tilde{x}, \tilde{y}$ by $x, y$ for convenience. The point $(1,1)$ will be moved into $(0,0)$ of the new system, and the system will be changed into the following system:

$$
\begin{aligned}
\frac{d x}{d \tau}= & 4 x^{2}-y-4 x y-3 x^{2} y+2 y^{2}+2 x y^{2} \\
& -\frac{\left(a_{20} x^{2}\right)}{\beta}-\frac{\left(a_{20} x^{3}\right)}{\beta}+\frac{\left(2 a_{20} x y\right)}{\beta} \\
& +\frac{\left(2 a_{20} x^{2} y\right)}{\beta}-\frac{\left(a_{20} y^{2}\right)}{\beta}-\frac{\left(a_{20} x y^{2}\right)}{\beta}+\frac{\left(2 x^{2} \theta\right)}{\beta} \\
& -\frac{(3 x y \theta)}{(2 \beta)}-\frac{\left(3 x^{2} y \theta\right)}{(2 \beta)}+\frac{\left(y^{2} \theta\right)}{(2 \beta)}+\frac{\left(x y^{2} \theta\right)}{(2 \beta)} \\
\frac{d y}{d \tau} & x-2 y^{2}-x y^{2}+2 y^{3}-\frac{\left(b_{20} x^{2}\right)}{\beta}+\frac{\left(2 b_{20} x y\right)}{\beta} \\
& -\frac{\left(b_{20} x^{2} y\right)}{\beta}-\frac{\left(b_{20} y^{2}\right)}{\beta}+\frac{\left(2 b_{20} x y^{2}\right)}{\beta}-\frac{\left(b_{20} y^{3}\right)}{\beta} \\
& +\frac{(x y \theta)}{(2 \beta)}-\frac{\left(3 y^{2} \theta\right)}{(2 \beta)}+\frac{\left(x y^{2} \theta\right)}{(2 \beta)}+\frac{\left(y^{3} \theta\right)}{(2 \beta)} .
\end{aligned}
$$

Furthermore, by the transformations

$$
\begin{array}{cl}
z=x+i y, & w=x-i y, \\
T=i \tau, & i=\sqrt{-1}
\end{array}
$$

system (4) can be transformed into the following system:

$$
\begin{aligned}
\frac{d z}{d T}=z+ & \frac{1}{8 \beta}\left(-2\left(2 a_{20}+2 i b_{20}-(6-2 i) \beta-(3-2 i) \theta\right) z^{2}\right. \\
& -2\left(-4 i a_{20}+4 b_{20}+(4+12 i) \beta+(3+5 i) \theta\right) z w \\
& +2\left(2 a_{20}+2 i b_{20}-(2+2 i) \beta-i \theta\right) w^{2} \\
& +\left(-2 a_{20}-2 b_{20}+(4+4 i) \beta+(1+i) \theta\right) z^{3} \\
& +2\left((-1+2 i) a_{20}+(1+2 i) b_{20}\right. \\
& +(1-4 i) \beta+(1-i) \theta) z^{2} w \\
& +\left((2+4 i) a_{20}+(2-4 i) b_{20}\right. \\
& -(1-i)(4 \beta+\theta)) z w^{2} \\
& \left.-2\left(-a_{20}+b_{20}+\beta+\theta\right)\right) w^{3}
\end{aligned}
$$

$$
\begin{aligned}
\frac{d w}{d T}=-w- & \frac{1}{8 \beta}\left(-2\left(2 a_{20}-2 i b_{20}-(6+2 i) \beta-(3+2 i) \theta\right) w^{2}\right. \\
& -2\left(4 i a_{20}+4 b_{20}+(4-12 i) \beta+(3-5 i) \theta\right) z w \\
& +2\left(2 a_{20}-2 i b_{20}-(2-2 i) \beta+i \theta\right) z^{2} \\
+ & \left(-2 a_{20}-2 b_{20}+(4-4 i) \beta+(1-i) \theta\right) w^{3} \\
+ & 2\left((-1-2 i) a_{20}+(1-2 i) b_{20}\right. \\
& +(1+4 i) \beta+(1+i) \theta) w^{2} z \\
+ & \left((2-4 i) a_{20}+(2+4 i) b_{20}\right. \\
& -(1+i)(4 \beta+\theta)) w z^{2} \\
& \left.-2\left(-a_{20}+b_{20}+\beta+\theta\right)\right) z^{3}
\end{aligned}
$$

applying the recursive formulae in Theorem 2.5 in [9], we compute singular point quantities and simplify them; then, we have the following theorem. 
Theorem 1. The first four singular point quantities at the origin of system (6) are as follows:

$$
\begin{aligned}
u_{1}= & \frac{i}{8 \beta^{2}}\left(-2 a_{20}-2 b_{20}+4 \beta+\theta\right) \\
& \times\left(-4 a_{20}+4 b_{20}+12 \beta+9 \theta\right), \\
u_{2}= & \frac{i}{192 \beta^{3}}\left(-2 a_{20}-2 b_{20}+4 \beta+\theta\right) \\
& \times\left(344 \beta^{2}+750 \beta \theta+325 \theta^{2}\right), \\
u_{3}=- & \frac{i}{12288 \beta^{5}}\left(-2 a_{20}-2 b_{20}+4 \beta+\theta\right) f_{1}, \\
u_{4}= & \frac{i}{5898240 \beta^{7}}\left(-2 a_{20}-2 b_{20}+4 \beta+\theta\right) f_{2},
\end{aligned}
$$

where

$$
\begin{aligned}
f_{1}= & -213760 b_{20}^{2} \beta^{2}-213760 b_{20} \beta^{3}+147392 \beta^{4} \\
& -323520 b_{20}^{2} \beta \theta-697600 b_{20} \beta^{2} \theta+84104 \beta^{3} \theta \\
& -116000 b_{20}^{2} \theta^{2}-682160 b_{20} \beta \theta^{2}-554530 \beta^{2} \theta^{2} \\
& -203000 b_{20} \theta^{3}-544505 \beta \theta^{3}-128125 \theta^{4} ; \\
f_{2}= & 14520162123939840 \beta^{10}+77088521496444928 \beta^{9} \theta \\
& +146627049158313472 \beta^{8} \theta^{2}+98147703728382592 \beta^{7} \theta^{3} \\
& -45397952769129120 \beta^{6} \theta^{4}-116390817537863400 \beta^{5} \theta^{5} \\
& -79417641637292700 \beta^{4} \theta^{6}-27193429543260550 \beta^{3} \theta^{7} \\
& -5139731868326875 \beta^{2} \theta^{8}-582232780375000 \beta \theta^{9} \\
& -38940972656250 \theta^{10} .
\end{aligned}
$$
holds.

While $u_{1}=u_{2}=u_{3}=0, u_{4} \neq 0$, the following theorem

Theorem 2. The origin of system (6) is a 4-order weak focus if and only if

$$
\begin{gathered}
a_{20}=\frac{1}{4}\left(4 b_{20}+12 \beta+9 \theta\right), \\
344 \beta^{2}+750 \beta \theta+325 \theta^{2}=0, \\
f_{1}=0 .
\end{gathered}
$$

Proof. $u_{1}=u_{2}=u_{3}=0$ implies that the relations above among parameters hold. Further, when $a_{20}=(1 / 4)\left(4 b_{20}+\right.$ $12 \beta+9 \theta), 344 \beta^{2}+750 \beta \theta+325 \theta^{2}=0$,

$$
\begin{aligned}
f_{1} & \\
= & -3164304640 b_{20}^{2}+94432128 \sqrt{1153} b_{20}^{2} \\
& -505511440 b_{20} \theta+16321424 \sqrt{1153} b_{20} \theta \\
& -1443860355 \theta^{2}+42746637 \sqrt{1153} \theta^{2} ; \\
f_{2} & \\
= & -748902307758080 b_{20}^{4}+22349440598016 \sqrt{1153} b_{20}^{4} \\
& -239280807055360 b_{20}^{3} \theta+7725648121856 \sqrt{1153} b_{20}^{3} \theta \\
& +5835163629391040 b_{20}^{2} \theta^{2}-171639395144768 \sqrt{1153} b_{20}^{2} \theta^{2} \\
& +969876044134880 b_{20} \theta^{3}-28776579351136 \sqrt{1153} b_{20} \theta^{3} \\
& +3030081181205925 \theta^{4}-89269930142715 \sqrt{1153} \theta^{4},
\end{aligned}
$$

we have

$$
\text { Resultant }\left[f_{1}, f_{2}, b_{20}\right]=2.87343 \times 10^{53} \theta^{8} \neq 0 \text {, }
$$

where Resultant $[g(x), f(x), x]$ denotes the resultant of $f(x), g(x)$ with respect to $x$. So $f_{2} \neq 0$ when $f_{1}=0$. Namely, point $(1,1)$ is a fourth-order weak focus.

We next study bifurcation of limit cycles of the perturbed system of (4). When condition (9) holds, we can obtain

$$
J=\left|\begin{array}{lll}
D\left[u_{1}, a_{20}\right] & D\left[u_{1}, \beta\right] & D\left[u_{1}, b_{20}\right] \\
D\left[u_{2}, a_{20}\right] & D\left[u_{2}, \beta\right] & D\left[u_{2}, b_{20}\right] \\
D\left[u_{3}, a_{20}\right] & D\left[u_{3}, \beta\right] & D\left[u_{3}, b_{20}\right]
\end{array}\right| \neq 0 .
$$

In fact,

$$
\begin{aligned}
J= & \frac{7001704448 i}{234375(-75+\sqrt{1153})^{11} \theta^{7}} \\
\times & \left(688 b_{20}+227 \theta+5 \sqrt{1153} \theta\right)^{2} \\
& \times\left(-17721692278080 b_{20}^{2}+519304637376 \sqrt{1153} b_{20}^{2}\right. \\
& \quad-2991374746080 b_{20} \theta+85097939808 \sqrt{1153} b_{20} \theta \\
& \left.\quad-2771947690805 \theta^{2}+80900776387 \sqrt{1153} \theta^{2}\right) .
\end{aligned}
$$

So Resultant $\left[J, f_{3}, b_{20}\right] \neq 0$, namely, $J \neq 0$, when $a_{20}=$ $(1 / 4)\left(4 b_{20}+12 \beta+9 \theta\right), 344 \beta^{2}+750 \beta \theta+325 \theta^{2}=0$.

The statement mentioned above yields that the following theorem holds.

Theorem 3. If the origin of system (4) is a 4-order weak focus, making a small perturbation to the coefficients of system (4), 
then, for perturbed system (4), in a small neighborhood of the origin, there exist exactly 5 small amplitude limit cycles enclosing the point $(1,1)$.

With a similar method, we could discuss the bifurcation of limit cycles from point $(2,2)$. Through the transformations $\tilde{x}=x-2, \tilde{y}=y-2, \tau=\theta t$, we still denote $\tilde{x}, \tilde{y}$ by $x, y$ for convenience. Then point $(1,1)$ will be moved into point $(0,0)$ of the new system, and the system will be changed into the following system:

$$
\begin{aligned}
\frac{d x}{d \tau}= & \frac{x^{2}}{2}-y-\frac{(7 x y)}{2}-\frac{\left(3 x^{2} y\right)}{2} \\
& +y^{2}+\frac{\left(x y^{2}\right)}{2}-\frac{\left(2 a_{20} x^{2}\right)}{\theta}-\frac{\left(a_{20} x^{3}\right)}{\theta} \\
& +\frac{\left(4 a_{20} x y\right)}{\theta}+\frac{\left(2 a_{20} x^{2} y\right)}{\theta}-\frac{\left(2 a_{20} y^{2}\right)}{\theta} \\
& -\frac{\left(a_{20} x y^{2}\right)}{\theta}+\frac{\left(x^{2} \beta\right)}{\theta}-\frac{(6 x y \beta)}{\theta}-\frac{\left(3 x^{2} y \beta\right)}{\theta} \\
& +\frac{\left(4 y^{2} \beta\right)}{\theta}+\frac{\left(2 x y^{2} \beta\right)}{\theta}, \\
\frac{d y}{d \tau}= & +\frac{(3 x y)}{2}+\frac{y^{2}}{2}+\frac{\left(x y^{2}\right)}{2}+\frac{y^{3}}{2} \\
& -\frac{\left(2 b_{20} x^{2}\right)}{\theta}+\frac{\left(4 b_{20} x y\right)}{\theta}-\frac{\left(b_{20} x^{2} y\right)}{\theta} \\
& -\frac{\left(2 b_{20} y^{2}\right)}{\theta}+\frac{\left(2 b_{20} x y^{2}\right)}{\theta}-\frac{\left(b_{20} y^{3}\right)}{\theta} \\
& -\frac{\left(3 y^{2} \beta\right)}{\theta}-\frac{\left(x y^{2} \beta\right)}{\theta}+\frac{\left(2 y^{3} \beta\right)}{\theta} .
\end{aligned}
$$

Furthermore, by the transformations

$$
z=x+i y, \quad w=x-i y, \quad T=i \tau, \quad i=\sqrt{-1},
$$

system (14) can be transformed into the following system:

$$
\begin{aligned}
\frac{d z}{d T}=z+ & \frac{1}{8 \theta}\left(2\left(-4 a_{20}-4 i b_{20}+(3+5 i) \beta+(3-i) \theta\right) z^{2}\right. \\
& +2\left(8 i a_{20}-8 b_{20}+(6-10 i) \beta+(1-3 i) \theta\right) z w \\
& -2\left(4 a_{20}+4 i b_{20}-(9-i) \beta-(4-2 i) \theta\right) w^{2} \\
& +\left(-2 a_{20}-2 b_{20}+4(1+4 i) \beta+(1+i) \theta\right) z^{3} \\
+ & 2\left((-1+2 i) a_{20}+(1+2 i) b_{20}\right. \\
& +(1-4 i) \beta+(1-i) \theta) z^{2} w \\
+ & \left(2(1+2 i) a_{20}+2(1-2 i) b_{20}-(1-i)(4 \beta+\theta)\right) z w^{2} \\
-2 & \left.\left(-a_{20}+b_{20}+\beta+\theta\right) w^{3}\right)
\end{aligned}
$$

$$
\begin{aligned}
\frac{d w}{d T}= & -w-\frac{1}{8 \theta}\left(2\left(-4 a_{20}+4 i b_{20}+(3-5 i) \beta+(3+i) \theta\right) w^{2}\right. \\
& +2\left(-8 i a_{20}-8 b_{20}+(6+10 i) \beta+(1+3 i) \theta\right) z w \\
& -2\left(4 a_{20}-4 i b_{20}-(9+i) \beta-(4+2 i) \theta\right) z^{2} \\
& +\left(-2 a_{20}-2 b_{20}+4(1-4 i) \beta+(1-i) \theta\right) w^{3} \\
& +2\left((-1-2 i) a_{20}+(1-2 i) b_{20}\right. \\
& +(1+4 i) \beta+(1+i) \theta) w^{2} z \\
& +\left(2(1-2 i) a_{20}+2(1+2 i) b_{20}-(1+i)(4 \beta+\theta)\right) w z^{2} \\
& \left.-2\left(-a_{20}+b_{20}+\beta+\theta\right) z^{3}\right) .
\end{aligned}
$$

Direct computation can give the following theorem.

Theorem 4. The first four singular point quantities at the origin of system (16) are as follows:

$$
\begin{aligned}
& u_{1}=\frac{i}{4 \theta^{2}}\left(-2 a_{20}-2 b_{20}+4 \beta+\theta\right)\left(8 a_{20}-8 b_{20}-3 \theta\right), \\
& u_{2}=-\frac{i}{48 \theta^{3}}\left(-2 a_{20}-2 b_{20}+4 \beta+\theta\right)\left(56 \beta^{2}+57 \beta \theta+10 \theta^{2}\right), \\
& u_{3}=\frac{i}{6144 \theta^{5}}\left(-2 a_{20}-2 b_{20}+4 \beta+\theta\right) f_{3}, \\
& u_{4}=-\frac{i}{5898240 \beta^{7}}\left(-2 a_{20}-2 b_{20}+4 \beta+\theta\right) f_{4},
\end{aligned}
$$

where

$$
\begin{aligned}
f_{3}= & -143360 b_{20}^{2} \beta^{2}+286720 b_{20} \beta^{3}-206080 \beta^{4} \\
& -168960 b_{20}^{2} \beta \theta+355840 b_{20} \beta^{2} \theta-264320 \beta^{3} \theta \\
& -40960 b_{20}^{2} \theta^{2}+103040 b_{20} \beta \theta^{2}-82336 \beta^{2} \theta^{2} \\
& +5120 b_{20} \theta^{3}-1939 \beta \theta^{3}+980 \theta^{4}, \\
f_{4}= & 2967207936000 \beta^{10}-9714651627520 \beta^{9} \theta \\
& -65013770321920 \beta^{8} \theta^{2}-124569363533824 \beta^{7} \theta^{3} \\
& -124914242976768 \beta^{6} \theta^{4}-75962577303936 \beta^{5} \theta^{5} \\
& -29760585599448 \beta^{4} \theta^{6}-7669544070125 \beta^{3} \theta^{7} \\
& -1275738647714 \beta^{2} \theta^{8}-125233021760 \beta \theta^{9} \\
& -5502919200 \theta^{10} .
\end{aligned}
$$

While solving $u_{1}=u_{2}=u_{3}=0$ and $u_{4} \neq 0$, we can immediately obtain the following theorem. 


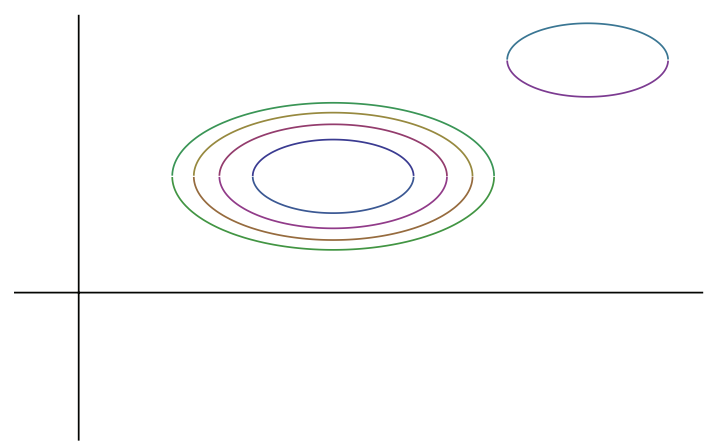

(a)

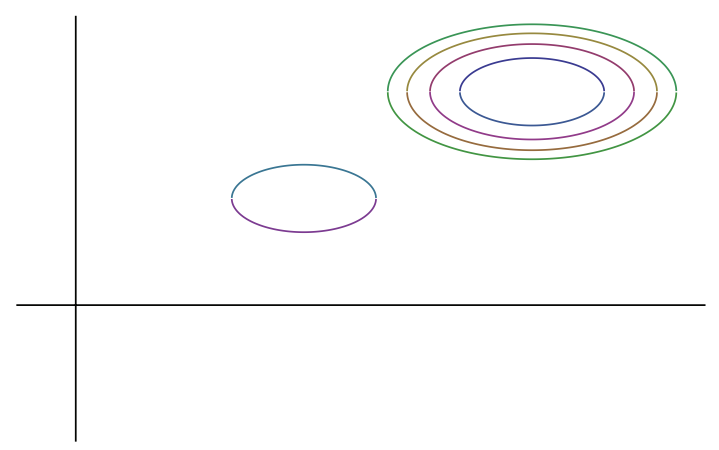

(b)

FIGURE 1: Distribution of limit cycles.

Theorem 5. The origin of system (16) is a 4-order weak focus if and only if

$$
\begin{gathered}
a_{20}=\frac{1}{8}\left(8 b_{20}+3 \theta\right), \quad 56 \beta^{2}+57 \beta \theta+10 \theta^{2}=0, \\
f_{3}=0 .
\end{gathered}
$$
(14).

Furthermore, the following theorem also holds for system

Theorem 6. If the origin of system (14) is a 4-order weak focus, making a small perturbation to the coefficients of system (14), then, for perturbed system (14), in a small neighborhood of the origin, there exist exactly 5 small amplitude limit cycles enclosing the point $(2,2)$.

The results also yield that when point $(1,1)$ is a fourthorder weak focus, point $(2,2)$ of system is a first-order weak focus at the same time. Point $(1,1)$ is a first-order weak focus when point $(2,2)$ is a fourth-order weak focus. Namely, by a simultaneously perturbation, five limit cycles could be bifurcated with two different distribution; see Figure 1.

\section{Center Conditions of Two Positive Equilibrium Points}

If all Lyapunov constants are equal to zero, it will be a center condition of a critical point. So Theorems 1 and 4 imply the following theorem.

Theorem 7. If the origins of systems (4) and (14) are centers if and only if

$$
a_{20}=\frac{1}{2}\left(-2 b_{20}+4 \beta+\theta\right) \text {. }
$$

Proof. When $a_{20}=(1 / 2)\left(-2 b_{20}+4 \beta+\theta\right)$, system (4) could be rewritten as

$$
\begin{aligned}
\frac{d x}{d \tau}=-y-\frac{1}{2 \beta} & \left(-2 b_{20} x^{2}-2 b_{20} x^{3}+4 b_{20} x y+4 b_{20} x^{2} y\right. \\
- & 2 b_{20} y^{2}-2 b_{20} x y^{2}-4 x^{2} \beta+4 x^{3} \beta
\end{aligned}
$$

$$
\begin{gathered}
\left.-2 x^{2} y \beta-3 x^{2} \theta+x^{3} \theta+x y \theta+x^{2} y \theta\right), \\
\frac{d y}{d \tau}=x-\frac{1}{2 \beta}\left(2 b_{20} x^{2}-4 b_{20} x y+2 b_{20} x^{2} y+2 b_{20} y^{2}\right. \\
-4 b_{20} x y^{2}+2 b_{20} y^{3}-2 x \beta+4 y^{2} \beta+2 x y^{2} \beta \\
\left.-4 y^{3} \beta-x y \theta+3 y^{2} \theta-x y^{2} \theta-y^{3} \theta\right) .
\end{gathered}
$$

By transformation

$$
x=-\frac{u+v}{2(v-1)}, \quad y=-\frac{-u+v}{2(v-1)}, \quad \tau=(v-1)^{2} t,
$$

system (21) becomes

$$
\begin{aligned}
& \frac{d u}{d \tau}=-v-\frac{1}{4 \beta}(-8 b_{20} u^{2}+4 b_{20} u^{4}+4 b_{20} u^{2} v-3 u^{4} \beta \\
&+ 4 v \beta+11 u^{2} v \beta-12 v^{2} \beta-9 u^{2} v^{2} \beta+9 v^{3} \beta \\
&-\left.4 u^{2} \beta+11 u^{2} v \theta-2 v^{2} \theta-8 u^{2} v^{2} \theta+3 v^{3} \theta\right), \\
& \frac{d v}{d \tau}=(u(-1+v)(-4 b_{20} u^{2}-4 \beta+3 u^{2} \beta \\
&\left.\left.+9 v^{2} \beta-6 v \theta+8 v^{2} \theta\right)\right) \times(4 \beta)^{-1}
\end{aligned}
$$

which is symmetric with $v$-axis.

When $a_{20}=(1 / 2)\left(-2 b_{20}+4 \beta+\theta\right)$, system (14) could be rewritten as

$$
\begin{aligned}
& \frac{d x}{d \tau} \\
& =-y-\frac{1}{2 \theta}\left(-4 b_{20} x^{2}-2 b_{20} x^{3}+8 b_{20} x y+4 b_{20} x^{2} y\right.
\end{aligned}
$$




$$
\begin{aligned}
& -4 b_{20} y^{2}-2 b_{20} x y^{2}+6 x^{2} \beta+4 x^{3} \beta-4 x y \beta \\
& \left.-2 x^{2} y \beta+x^{2} \theta+x^{3} \theta+3 x y \theta+x^{2} y \theta\right),
\end{aligned}
$$

$\frac{d y}{d \tau}$

$$
\begin{aligned}
=x+\frac{1}{2 \theta}( & -4 b_{20} x^{2}+8 b_{20} x y-2 b_{20} x^{2} y-4 b_{20} y^{2} \\
& +4 b_{20} x y^{2}-2 b_{20} y^{3}-4 x y \beta+6 y^{2} \beta \\
& \left.-2 x y^{2} \beta+4 y^{3} \beta+3 x y \theta+y^{2} \theta+x y^{2} \theta+y^{3} \theta\right) .
\end{aligned}
$$

Transformations

$$
x=-\frac{u+v}{2(v-1)}, \quad y=-\frac{-u+v}{2(v-1)}, \quad \tau=(v-1)^{2} t,
$$

bring system (24) into

$$
\begin{gathered}
\frac{d u}{d \tau} \\
=-v-\frac{1}{4 \theta}\left(-16 b_{20} u^{2}+4 b_{20} u^{4}+12 b_{20} u^{2} v\right. \\
+10 u^{2} \beta-3 u^{4} \beta-15 u^{2} v \beta+2 v^{2} \beta+7 u^{2} v^{2} \beta \\
\left.-v^{3} \beta+2 u^{2} \theta-7 u^{2} v \theta-4 v^{2} \theta+4 u^{2} v^{2} \theta+v^{3} \theta\right), \\
\frac{d v}{d \tau}=\left(u ( - 1 + v ) \left(4 b_{20} u^{2}-3 u^{2} \beta-12 v \beta\right.\right. \\
\left.\left.+7 v^{2} \beta+4 \theta-10 v \theta+4 v^{2} \theta\right)\right) \times(4 \theta)^{-1}
\end{gathered}
$$

which is symmetric with $v$-axis. So the origin is a center of system (14).

\section{Conflict of Interests}

The authors declare that they have no conflict of interests.

\section{Acknowledgments}

This research is partially supported by the National Nature Science Foundation of China (no. 11201211, 11201138, and 11261020) and the Scientific Research Fund of Hunan Provincial Education Department (no. 12B034), and Hunan Provincial Natural Science Foundation of China (no. 13JJ3106).

\section{References}

[1] X. S. Liu and Z. H. Zhuo, "Existence and uniqueness of limit cycles of a cubic Kolmogorov differential system," Biomathematics, vol. 3, pp. 266-271, 2001.

[2] X. C. Huang and L. Zhu, "Limit cycles in a general Kolmogorov model," Nonlinear Analysis: Theory, Methods and Applications, vol. 60, no. 8, pp. 1393-1414, 2005.
[3] Y. Ye and W. Ye, "Cubic Kolmogorov differential systems with two limit cycles surrounding the same focus," Annals of Differential Equations, vol. 2, pp. 201-207, 1985.

[4] E. Saez and I. Szanto, "Limit cycle s of a cubic Kolmogorov system," Applied Mathematics Letters, vol. 9, pp. 15-18, 1996.

[5] F. Li, "Integrability and bifurcations of limit cycles in a cubic Kolmogorov system," International Journal of Bifurcation and Chaos, vol. 23, no. 4, Article ID 1350061, 6 pages, 2013.

[6] C. Du and W. Huang, "Center-focus problem and limit cycles bifurcations for a class of cubic Kolmogorov model," Nonlinear Dynamics, vol. 72, no. 1-2, pp. 197-206, 2013.

[7] C. Du, Q. Wang, and W. Huang, "Three-dimensional Hopf bifurcation for a class of cubic Kolmogorov model," International Journal of Bifurcation and Chaos, vol. 24, no. 3, Article ID 1450036, 2014.

[8] C. Du, Y. Liu, and W. Huang, "Limit cycles bifurcations for a class of Kolmogorov model in symmetrical vector field," International Journal of Bifurcation and Chaos, vol. 24, no. 3, Article ID 1450040, 8 pages, 2014.

[9] Y. Liu and J. Li, "Theory of values of singular point in complex autonomous differential system," Science China A, vol. 3, pp. 245-255, 1989. 


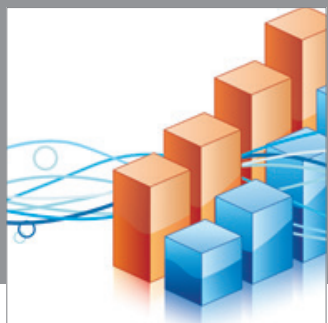

Advances in

Operations Research

mansans

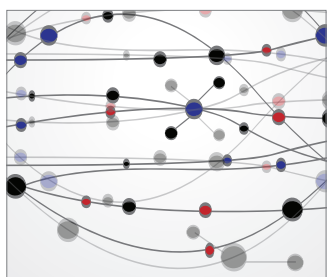

The Scientific World Journal
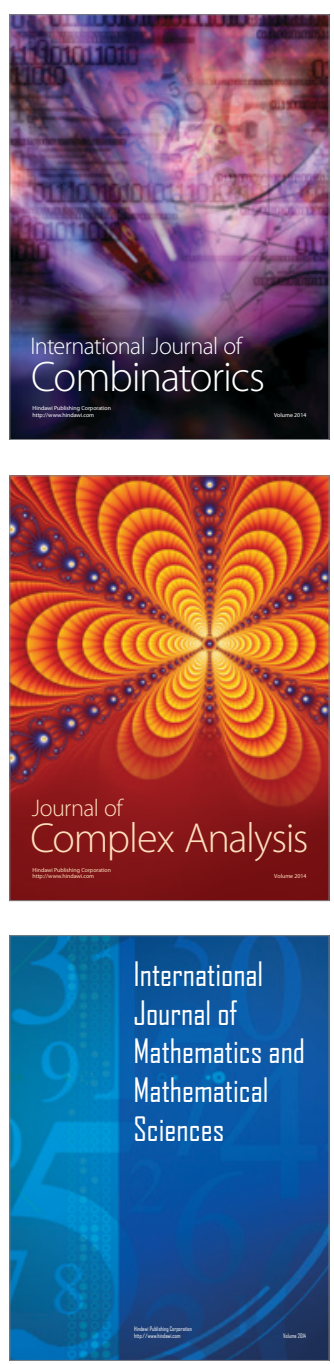
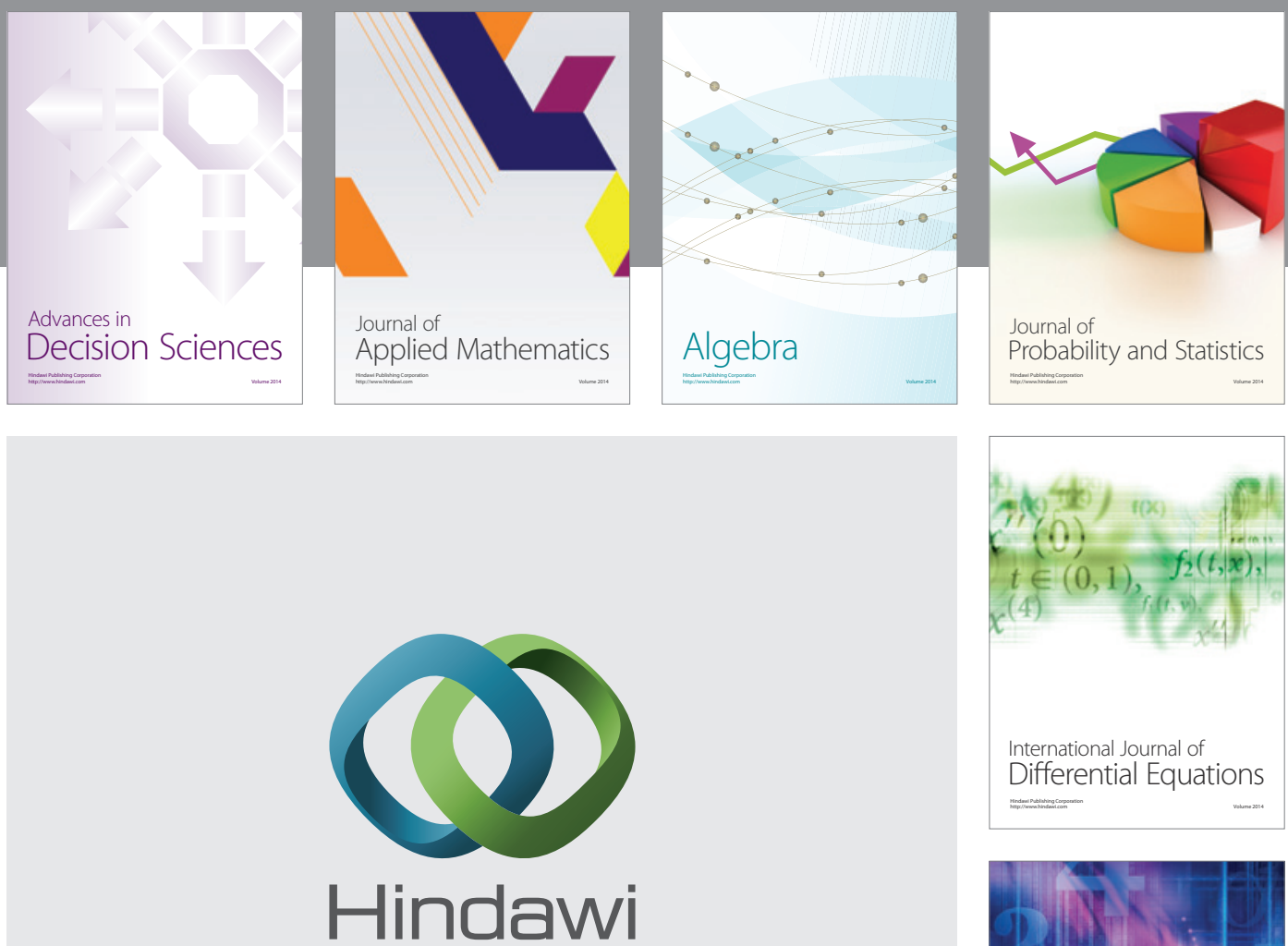

Submit your manuscripts at http://www.hindawi.com
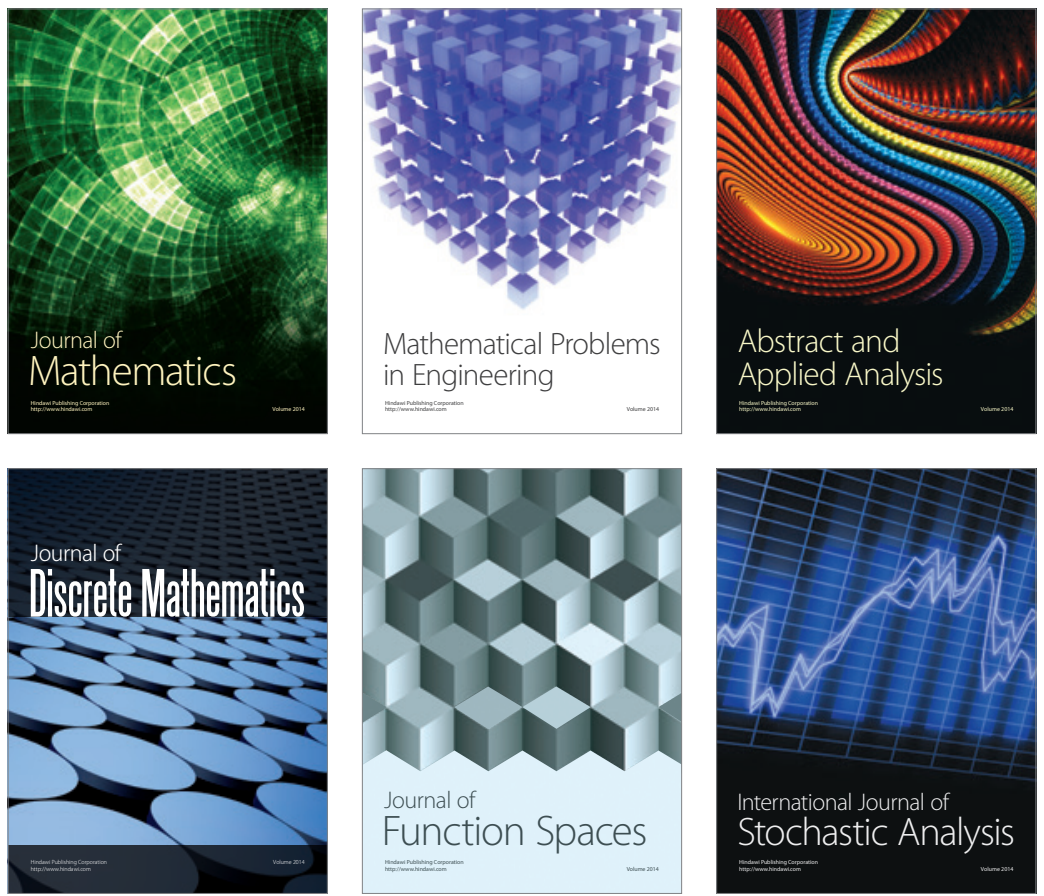

Journal of

Function Spaces

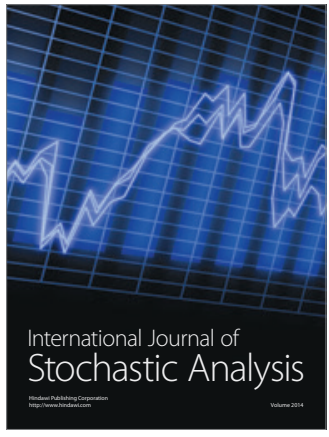

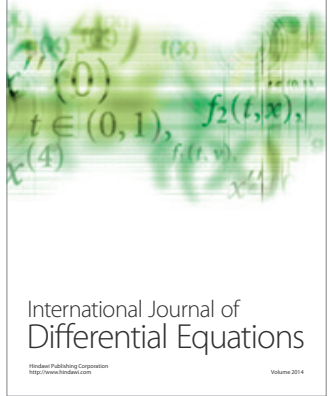
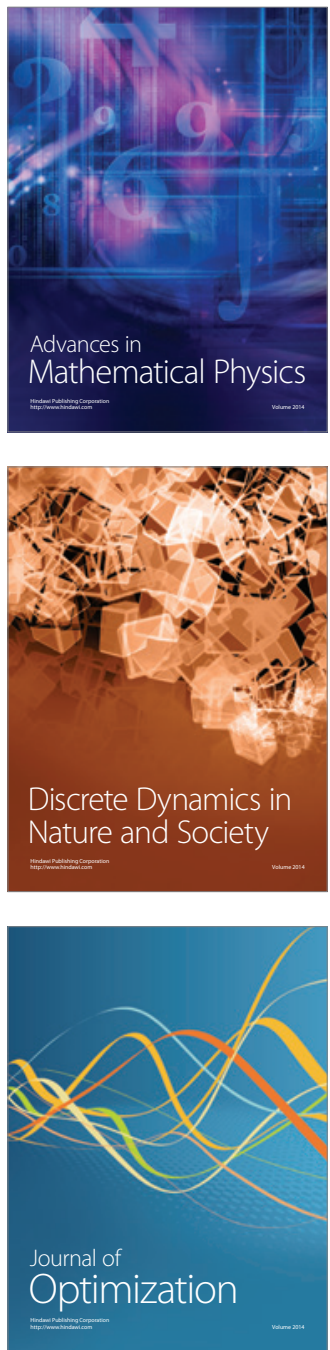